\title{
Precoded MDC-STBC Scheme With Half-Symbol Decodable Receiver
}

\author{
Hui Zhao ${ }^{1}$, Fanggang Wang ${ }^{1}$, Jinghua Kuang ${ }^{1}$, Wenbo Wang ${ }^{1}$ and Bin $\mathrm{Wu}^{2}$ \\ Key Lab of Universal Wireless Communications. Ministry of Education \\ ${ }^{1}$ Beijing University of Posts and Telecommunications, Beijing, P.R.China \\ ${ }^{2}$ Institute of Microelectronics of Chinese Academy of Sciences \\ Email: hzhao@bupt.edu.cn,wbwang@bupt.edu.cn
}

\begin{abstract}
It is well known that Space-Time Block Codes from orthogonal designs (O-STBC) are linearly MaximumLikelihood (ML) decodable. However there are not full rate complex O-STBC designs except for two transmit-antennas. Recently, one class of minimum-decoding-complexity STBCs (MDC-STBC) have been studied: (1) they have higher rate than O-STBC; (2) they are single-symbol decodable (SSD) and (3) they can exploit full diversity by constellation rotation (CR). In this paper, we employ precoding technology for these MDCSTBCs to realize: higher-rate, full-diversity, maximum coding gain and half-symbol decodable (HSD), where HSD has the lower complexity than SSD. Simulation results for MDCSTBCs with 4 transmit antennas show that the proposed precoder scheme with HSD decoder can get the same diversity gain and an extra 1dB coding gain than the schemes with $C R$, only at the cost of 3 bits feedback information for quantifying the precoder.
\end{abstract}

Key words: Quasi-orthogonal space-time block coding (QOSTBC), Minimum Decoding Complexity (MDC), SingleSymbol Decodable (SSD), precoding, feedback

\section{INTRODUCTION}

Coding for multiple-input multiple-output (MIMO) channels can significantly increase transmission reliability over wireless fading channels. For the system with two transmit antennas, the space-time block coding (STBC) scheme proposed by Alamouti is a noted full-diversity scheme, which has been employed in many communication systems (named as STTD in R99 of UMTS). For the system with more than two transmit antennas, Tarokh extended the theory of STBC in [1]. However the symbol-transmission rate of these orthogonal-STBCs (O-STBC) is smaller than one.

As to the system with four transmit antennas, a quasiorthogonal STBC (QO-STBC) with full rate is proposed in [2] that provide half of the maximum possible diversity and need maximum-likelihood (ML) detection to the pairs of transmitted symbols. And it was modified in [3] by constellation rotation (CR) to exploit the full diversity and in the meantime become real-symbol pair-wise ML decoding, which has same complexity as single-symbol decodable (SSD). Subsequently, coordinate interleaved orthogonal design (CIOD) has been proposed in [4] to also provide full diversity (after CR) and full rate with even SSD. Then the authors of [5-7] have proposed another kind of quasiorthogonal STBC independently. And the name of "minimum-decoding-complexity (MDC)" (same as SSD actually) happened to coincide. These schemes have the similar performance while the expression in $[5,7]$ is more concise so that the coding and decoding is a little simpler than that of [6]. In [8], Karmakar gave a comprehensive summary about MDC-STBCs and introduced their construction method based on Clifford algebras. Due to the property of full diversity, higher rate than O-STBC and SSD complexity, the MDC-STBCs should be readily applied to future wireless communication systems, such as beyond $3 \mathrm{G}$, IEEE $802.11 \mathrm{n}$ or $802.16 \mathrm{~m}$, when more than two transmit antennas are employed. The CIOD, which requires some of the transmit antennas to be turned off regularly so as to introduce high peak-to-average power ratio, and the other high rate STBCs based on cyclic division algebras with high decoding complexity are excluded in this paper. And only the square or rectangular constellations in common use are considered in the paper.

Precoding technology can improve the link reliability for STBCs, as discussed in [9]. Therefore, the precoder over STBC was designed to combat channel correlation in [10]. Many papers also studied the design criterion of precoding, such as minimization of pair-wise error probability in [11]. In [12], a multimode precoder design was investigated, which varies the number of streams depending on the channel condition. In this paper, we propose a novel precoding scheme for MDC-STBCs based on the particularity of the equivalent channel matrices of MDCSTBCs. Not only the performance can be improved, but also the decoding complexity will be lessened.

The rest of the paper is organized as follows. Section II gives the expanded real system model for linear dispersion STBC systems. After introducing the construction method of MDC-STBCs with arbitrary transmit antennas in Section III, a precoder scheme for these MDC-STBCs is proposed in Section IV. At the same time, its decoding complexity and performance are analyzed. Then, the simulation results for MDC-STBC with 4 transmit antennas in Section V show the proposed precoder with quantified feedback information is surely effective. Finally, conclusions are drawn in Section VI.

Notation: For a complex number $x, x^{R} x^{I}$ and $x^{*}$ denote its real part, imaginary part and conjugation respectively. 
$\mathbf{X}^{H}$ stands for the conjugated transposition of the matrix $\mathbf{X}$. $\mathbf{X}^{R}$ is the matrix composed of the real part of each element of $\mathbf{X}$, similarly, $\mathbf{X}^{I}$ composed of the imaginary part. The $n$ th row of $\mathbf{X}$ is denoted as $(\mathbf{X})_{n}$, and the $n$-th column of $\mathbf{X}$ as $[\mathbf{X}]_{n}$. The full-face $\mathbf{0}$ means zeros matrix.

\section{LINEAR DISPERSION STBC}

Suppose an MIMO system with $N_{t}$ transmit antennas and $N_{r}$ receive antennas, the channel is single-path and block fading in an interval of $T$ symbols. According to the introduced model in [13], the transmitted STC matrix can be written as a $N_{t} \times T$ matrix $\mathbf{S}$, which is composed of $Q$ complex symbols $s_{q}=s_{q}^{R}+j s_{q}^{I} \quad(q=1, \cdots, Q)$ and their dispersion matrices of size $N_{t} \times T\left\{\mathbf{A}_{q}, \mathbf{B}_{q}\right\}$ are:

$$
\mathbf{S}=\sum_{q=1}^{Q}\left(s_{q}^{R} \mathbf{A}_{q}+s_{q}^{I} \mathbf{B}_{q}\right)
$$

here $j=\sqrt{-1}$ is absorbed in $\mathbf{B}_{q}$. So the code rate of this linear dispersion $\mathrm{STBC}$ is defined as $R=Q / T$.

Then the system model can be expressed as:

$$
\mathbf{Y}=\mathbf{H S}+\mathbf{N}
$$

where $\mathbf{Y}$ is the received signal matrix, its element $y_{k, t}$ is the received symbol at the $k$-th receive antenna and the $t$-th interval; $\mathbf{H}$ is the channel matrix, and its element $h_{k, l}$ is the complex channel gain from the $l$-th transmit antenna to $k$-th receive antenna, which is independent complex Gaussian random variable with zero mean and unit variance; $\mathbf{N}$ is the noise matrix, and its element $n_{k, t}$ is a complex white Gaussian noise element with zero mean and covariance $\sigma_{n}^{2}$. Here, we omit the factor of power normalization before $\mathbf{S}$, which can be considered when calculating noise power for a given SNR.

Then we decompose each matrix into its real part and imaginary part:

$$
\begin{aligned}
& \mathbf{Y}^{R}=\sum_{q=1}^{Q}\left[\left(\mathbf{H}^{R} \mathbf{A}_{q}^{R}-\mathbf{H}^{I} \mathbf{A}_{q}^{I}\right) s_{q}^{R}+\left(\mathbf{H}^{R} \mathbf{B}_{q}^{R}-\mathbf{H}^{I} \mathbf{B}_{q}^{I}\right) s_{q}^{I}\right]+\mathbf{N}^{R} \\
& \mathbf{Y}^{I}=\sum_{q=1}^{Q}\left[\left(\mathbf{H}^{R} \mathbf{A}_{q}^{I}+\mathbf{H}^{I} \mathbf{A}_{q}^{R}\right) s_{q}^{R}+\left(\mathbf{H}^{R} \mathbf{B}_{q}^{I}+\mathbf{H}^{I} \mathbf{B}_{q}^{R}\right) s_{q}^{I}\right]+\mathbf{N}^{I}
\end{aligned}
$$

Let

$$
\begin{gathered}
\tilde{\mathbf{A}}_{q}=\left[\begin{array}{cc}
\mathbf{A}_{q}^{R} & \mathbf{A}_{q}^{I} \\
-\mathbf{A}_{q}^{I} & \mathbf{A}_{q}^{R}
\end{array}\right], \tilde{\mathbf{B}}_{q}=\left[\begin{array}{cc}
\mathbf{B}_{q}^{R} & \mathbf{B}_{q}^{I} \\
-\mathbf{B}_{q}^{I} & \mathbf{B}_{q}^{R}
\end{array}\right] \\
\underline{\mathbf{h}}_{l}=\left[\left(\mathbf{H}^{R}\right)_{l},\left(\mathbf{H}^{I}\right)_{l}\right]
\end{gathered}
$$

Therefore, the equation group of (3) can be rewritten as:

$$
\tilde{\mathbf{y}}=\tilde{\mathbf{H}} \tilde{\mathbf{x}}+\tilde{\mathbf{n}}
$$

where

$$
\tilde{\mathbf{H}}=\left[\begin{array}{ccccc}
\left(\underline{\mathbf{h}}_{1} \tilde{\mathbf{A}}_{1}\right)^{T} & \left(\underline{\mathbf{h}}_{1} \tilde{\mathbf{B}}_{1}\right)^{T} & \cdots & \left(\underline{\mathbf{h}}_{1} \tilde{\mathbf{A}}_{Q}\right)^{T} & \left(\underline{\mathbf{h}}_{1} \tilde{\mathbf{B}}_{Q}\right)^{T} \\
\vdots & \vdots & \ddots & \vdots & \vdots \\
\left(\underline{\mathbf{h}}_{N_{r}} \tilde{\mathbf{A}}_{1}\right)^{T} & \left(\underline{\mathbf{h}}_{N_{r}} \tilde{\mathbf{B}}_{1}\right)^{T} & \cdots & \left(\underline{\mathbf{h}}_{N_{r}} \tilde{\mathbf{A}}_{Q}\right)^{T} & \left(\underline{\mathbf{h}}_{N_{r}} \tilde{\mathbf{B}}_{Q}\right)^{T}
\end{array}\right]
$$

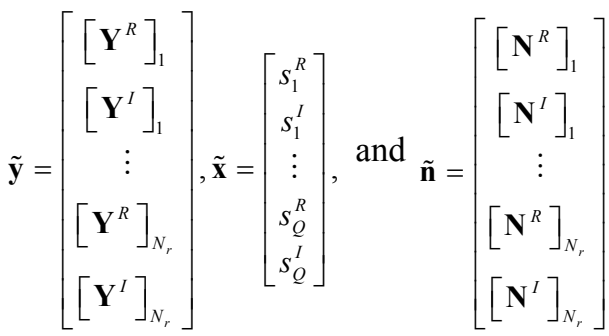

are the equivalent real channel matrix, received vector, transmitted vector and noise vector, respectively.

\section{CONSRUCTION OF MDC-STBC}

Before explaining our precoding scheme, we firstly introduce how to construct the needed MDC-STBC in this section. Firstly for the system with even transmit antennas, then with odd antennas.

\section{A. From O-STBC to MDC-STBC}

Assuming an O-STBC consists of $Q / 2$ sets of dispersion matrices denoted as $\left\{\underline{\mathbf{A}}_{p}, \underline{\mathbf{B}}_{p}\right\}(1 \leq p \leq Q / 2)$ for $N_{t} / 2$ transmit antennas with time interval $T / 2$. These $\left\{\underline{\mathbf{A}}_{p}, \underline{\mathbf{B}}_{p}\right\}$ have the following properties [8] (the following expressions are different from [5] because $j$ is not included in the dispersion matrices in (1) in [5]):

$$
\begin{aligned}
& \underline{\mathbf{A}}_{p}^{H} \underline{\mathbf{A}}_{p}=\underline{\mathbf{B}}_{p}^{H} \underline{\mathbf{B}}_{p}=\mathbf{I}_{N_{t} / 2} \\
& \underline{\mathbf{A}}_{p_{1}}^{H} \underline{\mathbf{A}}_{p_{2}}+\underline{\mathbf{A}}_{p_{2}}^{H} \underline{\mathbf{A}}_{p_{1}}=0, \underline{\mathbf{B}}_{p_{1}}^{H} \underline{\mathbf{B}}_{p_{2}}+\underline{\mathbf{B}}_{p_{2}}^{H} \underline{\mathbf{B}}_{p_{1}}=0\left(p_{1} \neq p_{2}\right) \\
& \underline{\mathbf{A}}_{p_{1}}^{H} \underline{\mathbf{B}}_{p_{2}}+\underline{\mathbf{B}}_{p_{2}}^{H} \underline{\mathbf{A}}_{p_{1}}=0\left(p_{1} \neq p_{2}\right)
\end{aligned}
$$

Here, only (7-ii) and (7-iii) are the sufficient and necessary condition for an O-STBC. Generally, unitary weighting (UW) can simplify matrix multiplication and keep lower PAPR. So we discuss the UW-STBC and the condition (1) appears.

According to $[5,8]$, the dispersion matrices of O-STBC can be used to build an MDC-STBC, which consists of $Q$ sets of dispersion matrices $\left\{\mathbf{A}_{q}, \mathbf{B}_{q}\right\}(1 \leq q \leq Q)$ for $N_{t}$ transmit antennas with interval $T$, when the following mapping rules are satisfied:

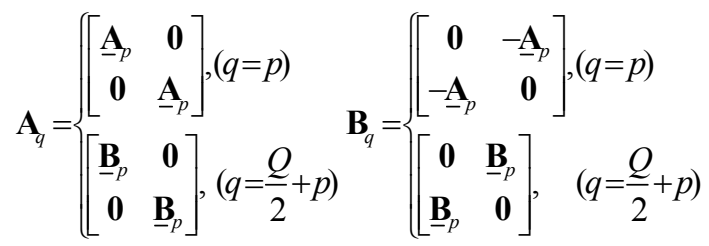

Using (7) and (8), it is easy to prove that $\left\{\mathbf{A}_{q}, \mathbf{B}_{q}\right\}$ satisfy the following properties (Generally, there must be an identity matrix in $\left\{\mathbf{A}_{q}, \mathbf{B}_{q}\right\}$, so we let $\left.\mathbf{A}_{1}=\mathbf{I}\right)$ :

$$
\begin{aligned}
& \mathbf{A}_{q}^{H}=-\mathbf{A}_{q}(q \geq 2) \\
& \mathbf{A}_{q_{1}}^{H} \mathbf{A}_{q_{2}}+\mathbf{A}_{q_{2}}^{H} \mathbf{A}_{q_{1}}=0\left(q_{1} \neq q_{2}\right) \\
& \mathbf{B}_{p}^{H}=\mathbf{B}_{p} \\
& \mathbf{B}_{1} \mathbf{A}_{q}=\mathbf{A}_{q} \mathbf{B}_{1} \\
& \mathbf{B}_{q}= \pm \mathbf{B}_{1} \mathbf{A}_{q}(q \geq 2)
\end{aligned}
$$


Those are the sufficient condition for a linear dispersion STBC to become an MDC scheme. When these conditions are all satisfied, it is easy to demonstrate [5] the correlation of the equivalent channel matrix $\tilde{\mathbf{H}}$ in (6) is symmetric block-diagonal with nonzero submatrices of size $2 \times 2$, like the form in (10).

$$
\tilde{\mathbf{H}}^{T} \tilde{\mathbf{H}}=\left[\begin{array}{cccc}
\cdots & \cdots & \cdots & \cdots \\
\vdots & \sum_{l=1}^{N_{r}} \underline{\mathbf{h}}_{l} \tilde{\mathbf{A}}_{q} \tilde{\mathbf{A}}_{q}^{T} \underline{\mathbf{h}}_{l}^{T} & \sum_{l=1}^{N_{r}} \underline{\mathbf{h}}_{l} \tilde{\mathbf{A}}_{q} \tilde{\mathbf{B}}_{q}^{T} \underline{\mathbf{h}}_{l}^{T} & \vdots \\
\vdots & \sum_{l=1}^{N_{r}} \underline{\mathbf{h}}_{l} \tilde{\mathbf{A}}_{q} \tilde{\mathbf{B}}_{q}^{T} \underline{\mathbf{h}}_{l}^{T} & \sum_{l=1}^{N_{r}} \underline{\mathbf{h}}_{l} \tilde{\mathbf{A}}_{q} \tilde{\mathbf{A}}_{q}^{T} \underline{\mathbf{h}}_{l}^{T} & \vdots \\
\cdots & \cdots & \cdots & \ldots
\end{array}\right]_{2 Q \times 2 Q}
$$

After the matched filtering at the receiver, the diagonal element in $\tilde{\mathbf{H}}^{T} \tilde{\mathbf{H}}$ is regarded as the signal power for each element in $\tilde{\mathbf{x}}$ and the non-diagonal element as ISI. Then the minimization of ML decoding metric of (6) can be decomposed as the minimization of the $\mathrm{Q}$ independent metrics, where each is corresponding to a single complex symbol decoupled with the others, namely,

$$
\min _{s_{1}, \cdots, s_{Q}}\|\tilde{\mathbf{y}}-\tilde{\mathbf{H}} \tilde{\mathbf{x}}\|^{2} \Leftrightarrow \min _{s_{q}} f_{q}\left(s_{q}\right),(1 \leq q \leq Q)
$$

That is why MDC is also called SSD.

\section{B. MDC-STBC with Odd Transmit Antennas}

As we all known, a complex O-STBC design exists only if $N_{t}=2$ and 4 [1] and the code rates are 1 and $3 / 4$ respectively. By the construction equation (8), we obtain the MDC-STBC with even transmit antennas $N_{t}=4$ and 8 . The constructed complex MDC-STBCs have a code rate, which is same as the half-size O-STBCs used to construct it. On the other hand, for odd transmit antennas $N_{t}^{o}$ that can be generated by removing some rows of the space-time coding matrix with a smallest even transmit antennas $N_{t}^{e}$, which satisfies $N_{t}^{o}<N_{t}^{e}=2^{n}$ ( $n$ is an integer). This keeps the characteristics of MDC-STBC and the same code rate except the diversity gain due to the lack of transmit antennas.

Therefore, we can conclude the MDC-STBCs for the systems with $N_{t}=3 \sim 8$ have a higher code rate than corresponding O-STBCs. The gap is shown in the Fig.1.It is noted that, in this figure, the O-STBC and MDC-STBC for a certain $N_{t}$ has the minimum coding period $\left(T<2 N_{t}\right)$. There exist some other complex orthogonal designs [14] with the higher rate than [1] but the longer $T$, which needs the static channel with long period so as to be infeasible. Now, for the systems with $N_{t}=3 \sim 8$, there are practical MDCSTBC schemes. These space-time matrices are easy to write by (8).

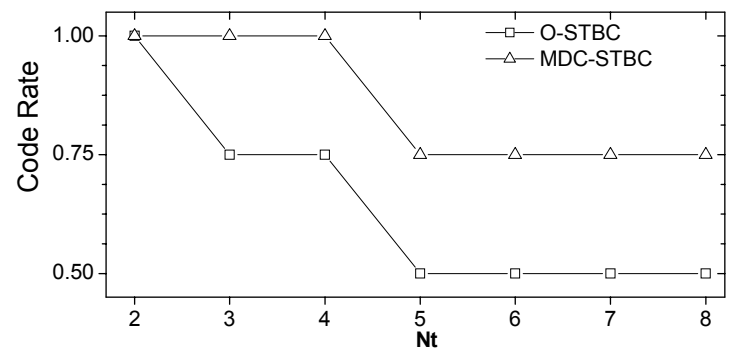

Figure 1. The comparison of code rate between O-STBC and MDC-STBC

\section{PRECODING FOR MDC-STBCS}

In this section, we will explain how to lessen the detection complexity by precoding for MDC-STBCs.

\section{A. The Expression of Interfrence Items}

Here, we discuss a different decoding algorithm with that in [5]. At the receiver, a matched filter $\tilde{\mathbf{H}}^{T}$ is left-multiplied to $\tilde{\mathbf{y}}$ in (6), then the system model (6) can be modified as:

$$
\tilde{\mathbf{H}}^{T} \tilde{\mathbf{y}}=\tilde{\mathbf{H}}^{T} \tilde{\mathbf{H}} \tilde{\mathbf{x}}+\tilde{\mathbf{H}}^{T} \tilde{\mathbf{n}}
$$

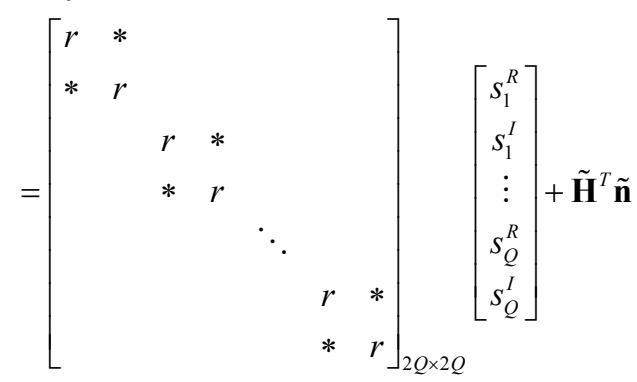

where $r=\sum_{l=1}^{N_{r}} \underline{\mathbf{h}}_{l} \tilde{\mathbf{A}}_{q} \tilde{\mathbf{A}}_{q}^{T} \underline{\mathbf{h}}_{l}^{T}$ and $*=\sum_{i=1}^{N_{r}} \underline{\mathbf{h}}_{i} \tilde{\mathbf{A}}_{q} \tilde{\mathbf{B}}_{q}^{T} \underline{\mathbf{h}}_{i}^{T}$

According to the definition of (4) and the property of (9), we know

$$
\begin{aligned}
\tilde{\mathbf{A}}_{q}^{\tilde{\mathbf{A}}_{q}^{T}} & =\left[\begin{array}{cc}
\mathbf{A}_{q}^{R} & \mathbf{A}_{q}^{I} \\
-\mathbf{A}_{q}^{I} & \mathbf{A}_{q}^{R}
\end{array}\right]\left[\begin{array}{cc}
\mathbf{A}_{q}^{R} & \mathbf{A}_{q}^{I} \\
-\mathbf{A}_{q}^{I} & \mathbf{A}_{q}^{R}
\end{array}\right]^{T} \\
& =\left[\begin{array}{cc}
\left(\mathbf{A}_{q} \mathbf{A}_{q}^{H}\right)^{R} & \left(\mathbf{A}_{q} \mathbf{A}_{q}^{H}\right)^{I} \\
-\left(\mathbf{A}_{q} \mathbf{A}_{q}^{H}\right)^{I} & \left(\mathbf{A}_{q} \mathbf{A}_{q}^{H}\right)^{R}
\end{array}\right]=\left[\begin{array}{cc}
\mathbf{I}_{N t} & \mathbf{0} \\
\mathbf{0} & \mathbf{I}_{N t}
\end{array}\right] \\
\tilde{\mathbf{A}}_{q} \tilde{\mathbf{B}}_{q}^{T} & =\left[\begin{array}{cc}
\mathbf{A}_{q}^{R} & \mathbf{A}_{q}^{I} \\
-\mathbf{A}_{q}^{I} & \mathbf{A}_{q}^{R}
\end{array}\right]\left[\begin{array}{cc}
\mathbf{B}_{q}^{R} & \mathbf{B}_{q}^{I} \\
-\mathbf{B}_{q}^{I} & \mathbf{B}_{q}^{R}
\end{array}\right]^{T} \\
& =\left[\begin{array}{cc}
\left(\mathbf{A}_{q} \mathbf{B}_{q}^{H}\right)^{R} & \left(\mathbf{A}_{q} \mathbf{B}_{q}^{H}\right)^{I} \\
-\left(\mathbf{A}_{q} \mathbf{B}_{q}^{H}\right)^{I} & \left(\mathbf{A}_{q} \mathbf{B}_{q}^{H}\right)^{R}
\end{array}\right] \stackrel{(-v)}{=}\left[\begin{array}{ll}
\left( \pm \mathbf{B}_{1}\right)^{R} & \left( \pm \mathbf{B}_{1}\right)^{I} \\
\left(\mp \mathbf{B}_{1}\right)^{I} & \left( \pm \mathbf{B}_{1}\right)^{R}
\end{array}\right]
\end{aligned}
$$

Then the elements of the diagonal-block matrix of $\tilde{\mathbf{H}}^{T} \tilde{\mathbf{H}}$ are:

$$
\begin{gathered}
r=\sum_{l=1}^{N_{r}} \underline{\mathbf{h}}_{l} \underline{\mathbf{h}}_{l}^{T}=\sum_{k=1}^{N_{r}} \sum_{l=1}^{N_{t}}\left|h_{k, l}\right|^{2} \\
*=\sum_{l=1}^{N_{r}} \underline{\mathbf{h}}_{l}\left[\begin{array}{ll}
\left( \pm \mathbf{B}_{1}\right)^{R} & \left( \pm \mathbf{B}_{1}\right)^{I} \\
\left(\mp \mathbf{B}_{1}\right)^{I} & \left( \pm \mathbf{B}_{1}\right)^{R}
\end{array}\right] \underline{\mathbf{h}}_{l}^{T}
\end{gathered}
$$

Obviously, $r$ represents the efficient signal power, “*” is the 
interference item between a pair of real symbols $\left(s_{q}^{R}, s_{q}^{I}\right)$, and its value depends on the choice of the dispersion matrices for each symbol. It is noted the "*" in different block may have the different signs but the same absolute value.

For an intuitionistic explanation, we take two typical examples of MDC-STBC with $N_{t}=4$. One is given in [5]. The space-time code is

$$
\mathbf{S}_{1}=\left(\begin{array}{cccc}
s_{1}^{R}+j s_{3}^{R} & s_{2}^{R}+j s_{4}^{R} & -s_{1}^{I}+j s_{3}^{I} & -s_{2}^{I}+j s_{4}^{I} \\
-s_{2}^{R}+j s_{4}^{R} & s_{1}^{R}-j s_{3}^{R} & s_{2}^{I}+j s_{4}^{I} & -s_{1}^{I}-j s_{3}^{I} \\
-s_{1}^{I}+j s_{3}^{I} & -s_{2}^{I}+j s_{4}^{I} & s_{1}^{R}+j s_{3}^{R} & s_{2}^{R}+j s_{4}^{R} \\
s_{2}^{I}+j s_{4}^{I} & -s_{1}^{I}-j s_{3}^{I} & -s_{2}^{R}+j s_{4}^{R} & s_{1}^{R}-j s_{3}^{R}
\end{array}\right)
$$

Now, the correlation matrix of its equivalent channel matrix can be written as:

$$
\tilde{\mathbf{H}}_{1}^{T} \tilde{\mathbf{H}}_{1}=\left[\begin{array}{cccccccc}
r & a & & & & & & \\
a & r & & & & & & \\
& & r & a & & & & \\
& & a & r & & & & \\
& & & & r & -a & & \\
& & & & -a & r & & \\
& & & & & & r & a \\
& & & & & & a & r
\end{array}\right]
$$

where $r=\sum_{k=1}^{N_{r}} \sum_{l=1}^{4}\left|h_{k, l}\right|^{2}$ and $a=-2 \sum_{k=1}^{N_{r}}\left\{h_{k, 1}^{*} h_{k, 3}+h_{k, 2} h_{k, 4}^{*}\right\}^{R}$.

The other example is given in [8], which space-time coding matrix is:

$$
\mathrm{S}_{2}=\left(\begin{array}{cccc}
s_{1}^{R}-j s_{4}^{I} & s_{2}^{R}+j s_{3}^{R} & s_{4}^{R}+j s_{1}^{I} & -s_{3}^{I}+j s_{2}^{I} \\
-s_{2}^{R}+j s_{3}^{R} & s_{1}^{R}+j s_{4}^{I} & -s_{3}^{I}-j s_{2}^{I} & -s_{4}^{R}+j s_{1}^{I} \\
-s_{4}^{R}-j s_{1}^{I} & s_{3}^{I}-j s_{2}^{I} & s_{1}^{R}-j s_{4}^{I} & s_{2}^{R}+j s_{3}^{R} \\
s_{3}^{I}+j s_{2}^{I} & s_{4}^{R}-j s_{1}^{I} & -s_{2}^{R}+j s_{3}^{R} & s_{1}^{R}+j s_{4}^{I}
\end{array}\right)
$$

Similarly, the correlation matrix of its equivalent channel matrix is

$$
\tilde{\mathbf{H}}_{2}^{T} \tilde{\mathbf{H}}_{2}=\left[\begin{array}{llllllll}
r & b & & & & & \\
b & r & & & & & & \\
& & r & b & & & & \\
& & b & r & & & & \\
& & & & r & b & & \\
& & & & b & r & & \\
& & & & & & r & b \\
& & & & & b & r
\end{array}\right]
$$

where $r=\sum_{k=1}^{N_{r}} \sum_{l=1}^{4}\left|h_{k, l}\right|^{2}$ and $b=-2 \sum_{k=1}^{N_{r}}\left\{h_{k, 1}^{*} h_{k, 3}-h_{k, 2} h_{k, 4}^{*}\right\}^{I}$.

Although the values of $a$ and $b$ are different, their statistical properties are same when the channel coefficients are independent one another. Then the channel correlation matrix (16) and (18) clarify that these two schemes should have the same performance.

Assuming all the dispersion matrices are chosen from the Clifford matrices [8], the elements of these dispersion matrices are chosen from $\{ \pm 1, \pm j, 0\}$. Moreover, \pm 1 and $\pm j$ do not appear in a matrix at the same time. Now, by observing the two interference items, the absolute value of the interference item has the following expression:

$$
|*|=2 \sum_{k=1}^{N_{r}} \sum_{l_{1}=1, l_{2} \neq l_{1}}^{N_{t} / 2}\left\{ \pm h_{k, l_{1}}^{*} h_{k, l_{2}}\right\}^{\text {Ror I }}
$$

where $1 \leq l_{1} \neq l_{2} \leq N_{t}$. Here whether the value is the real part or imaginary part of $\left\{ \pm h_{k, l_{1}}^{*} h_{k, l_{2}}\right\}$ depends on the choice of $\mathbf{A}_{q}$ and $\mathbf{B}_{q}$, according to (13-ii). When all the $\mathbf{B}_{q}$ are composed of $\{ \pm j, 0\}$, it is the imaginary part; otherwise, it is the real part.

\section{B. Precoding Design}

Now, we design the precoder $\mathbf{P}$ for MDC-STBCs with Clifford matrices as the dispersion matrices, then the system model (2) will be changed as:

$$
\mathbf{Y}^{\prime}=\mathbf{H P S}+\mathbf{N}=\mathbf{H}^{\prime} \mathbf{S}+\mathbf{N}
$$

Due to the issues of PAPR, suppose $\mathbf{P}$ is a unimodular diagonal matrix, which has the form as:

$$
\mathbf{P}=\left[\begin{array}{cccc}
e^{j \theta_{1}} & 0 & \cdots & 0 \\
0 & e^{j \theta_{2}} & \cdots & 0 \\
\vdots & \vdots & \ddots & \vdots \\
0 & 0 & \cdots & e^{j \theta_{N t}}
\end{array}\right]
$$

Then the $l$-th column of the new generalized channel matrix is $\left[\mathbf{H}^{\prime}\right]_{l}=e^{j \theta_{l}}[\mathbf{H}]_{l}$. Now, the interference after precoding is

$$
|*|=2 \sum_{k=1}^{N_{r}} \sum_{l_{1}=1, l_{2} \neq l_{1}}^{N_{t} / 2}\left\{ \pm e^{j \theta_{l_{1}}} h_{k, l_{1}}^{*} e^{j \theta_{l_{2}}} h_{k, l_{2}}\right\}^{I \text { or } R}
$$

To forcing the interference item to zero, we let

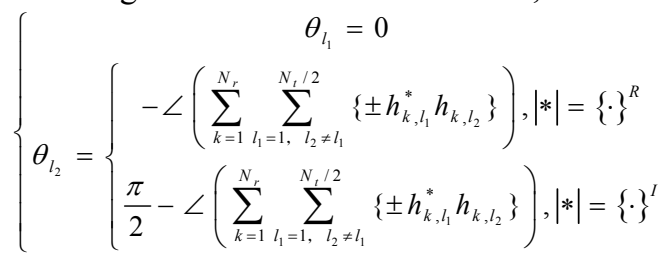

\section{Analysis of complexity}

Then, after adding the precoder, (12) will become into:

$$
\begin{aligned}
& \tilde{\mathbf{H}}^{\prime T} \tilde{\mathbf{y}}=\tilde{\mathbf{H}}^{\prime T} \tilde{\mathbf{H}}{ }^{\prime} \tilde{\mathbf{x}}+\tilde{\mathbf{H}}^{\prime T} \tilde{\mathbf{n}} \\
& =\left[\begin{array}{ccccccc}
r & 0 & & & & & \\
0 & r & & & & & \\
& & r & 0 & & & \\
& & 0 & r & & & \\
& & & & \ddots & & \\
& & & & & r & 0 \\
& & & & & 0 & r
\end{array}\right]_{2 Q \times 2 Q}\left[\begin{array}{c}
s_{1}^{R} \\
s_{1}^{I} \\
\vdots \\
s_{Q}^{R} \\
s_{Q}^{I}
\end{array}\right]+\tilde{\mathbf{H}}^{\prime T} \tilde{\mathbf{n}}
\end{aligned}
$$

We found the new equivalent channel correlation matrix after precoding will become a pure diagonal matrix, which the diagonal element is $r$. Now the decoding for (24) will be half-symbol decodable (HSD). Namely the ML decoding metric can be decomposed as the $2 Q$ independent metrics: 
TABLE I. COMPARISON BETWEEN HSD AND SSD

\begin{tabular}{|l|c|c|c|c|}
\hline & QPSK & 16QAM & 64QAM & 256QAM \\
\hline HSD & $2 \times 2=4$ & $4 \times 2=8$ & $8 \times 2=16$ & $16 \times 2=32$ \\
\hline SSD & 4 & 16 & 64 & 256 \\
\hline ratio & $100 \%$ & $50 \%$ & $25 \%$ & $12.5 \%$ \\
\hline
\end{tabular}

$\min _{s_{1}, \cdots, s_{Q}}\left\|\tilde{\mathbf{H}}^{\prime T} \tilde{\mathbf{y}}-\tilde{\mathbf{H}}^{\prime T} \tilde{\mathbf{H}} \tilde{\mathbf{x}}^{\prime}\right\|^{2} \Leftrightarrow\left\{\begin{array}{l}\min _{s_{q}^{R}}\left\|\left(\tilde{\mathbf{H}}^{\prime T} \tilde{\mathbf{y}}\right)_{2 q-1}-r s_{q}^{R}\right\|^{2} \\ \min _{s_{q}^{l}}\left\|\left(\tilde{\mathbf{H}}^{T} \tilde{\mathbf{y}}\right)_{2 q}-r s_{q}^{I}\right\|^{2}\end{array}(1 \leq q \leq Q)\right.$

When the modulation level is high, the advantage of low complexity for HSD is distinct comparing with SSD. In Table I, they are measured by the search times for estimating a complex symbol. Because of the decoupling of real part and imaginary part, HSD only do searching in a smaller search space, which ratio to that of SSD is exponentially decreased with the increase of modulation level.

\section{Analysis of performance}

For the system model (20), it ML decoding metric should be

$$
\min _{s_{1}, \cdots, s_{Q}}\left\|\mathbf{Y}^{\prime}-\mathbf{H}^{\prime} \mathbf{S}\right\|^{2}
$$

Now, observing the used matched filter $\tilde{\mathbf{H}}^{\prime T}$, due to $\tilde{\mathbf{H}}^{\prime T} \tilde{\mathbf{H}}^{\prime}=r \cdot \mathbf{I}_{2 Q}, \tilde{\mathbf{H}}^{\prime T}$ should be an orthogonal matrix, in which each column has the same norm $r$ and is orthogonal with one another. Then according to the matrix theory [15], the following relation is satisfied:

$$
\left\|\tilde{\mathbf{H}}^{\prime T}\right\|^{2}\left\|\tilde{\mathbf{y}}-\tilde{\mathbf{H}}^{\prime} \tilde{\mathbf{x}}\right\|^{2}=\left\|\tilde{\mathbf{H}}^{\prime T}\left(\tilde{\mathbf{y}}-\tilde{\mathbf{H}} \tilde{\mathbf{x}}^{\prime}\right)\right\|^{2}=\left\|\tilde{\mathbf{H}}^{\prime T} \tilde{\mathbf{y}}-\tilde{\mathbf{H}}^{\prime T} \tilde{\mathbf{H}}{ }^{\prime} \tilde{\mathbf{x}}\right\|^{2}
$$

So we can derive the relation between (26) and (25):

$$
\begin{aligned}
& \min _{s_{1}, \cdots, s_{Q}}\left\|\mathbf{Y}^{\prime}-\mathbf{H}^{\prime} \mathbf{S}\right\|^{2} \\
& \Leftrightarrow \min _{s_{1}, \cdots, s_{Q}} r^{2 Q} \cdot\left\|\tilde{\mathbf{y}}-\tilde{\mathbf{H}} \tilde{\mathbf{x}}^{\prime}\right\|^{2}=\min _{s_{1}, \cdots, s_{Q}}\left\|\tilde{\mathbf{H}}^{\prime T}\right\|^{2}\left\|\tilde{\mathbf{y}}-\tilde{\mathbf{H}}{ }^{\prime} \tilde{\mathbf{x}}\right\|^{2} \\
& =\min _{s_{1}, \cdots, s_{Q}}\left\|\tilde{\mathbf{H}}^{\prime T} \tilde{\mathbf{y}}-\tilde{\mathbf{H}}^{\prime T} \tilde{\mathbf{H}}^{\prime} \tilde{\mathbf{x}}\right\|^{2}
\end{aligned}
$$

That is to say, this algorithm of decoding the filtered system model (24) is equivalent with that of decoding the original system model (20) using ML criterion. Namely, (25) is the optimal solution of (20), although we use the different decoding with [5]. It is noted that this conclusion is right only if the matched filter matrix is orthogonal.

Obviously, it is the specific precoder design that makes the matched filter matrix orthogonal. And due to the zeroforcing interference between the pair of real symbols, the precoder will improve the link performance, comparing with the system without precoder.

\section{E. Examples}

In the same way, we give some examples of the exact precoder matrix.

For $\mathrm{S}_{1}$ and $\mathrm{S}_{2}$ in subsection $\mathrm{A}$, they may use the precoder with the same form:
$\mathbf{P}=\left[\begin{array}{llll}1 & & & \\ & 1 & & \\ & & e^{j \theta} & \\ & & & e^{j \theta}\end{array}\right]$ where $\theta=-\angle\left(\sum_{k=1}^{N_{r}}\left(h_{k, 1}^{*} h_{k, 3}+h_{k, 2}^{*} h_{k, 4}\right)\right)$

for $\mathrm{S}_{1}$ and $\theta=\frac{\pi}{2}-\angle\left(\sum_{k=1}^{N_{r}}\left(h_{k, 1}^{*} h_{k, 3}-h_{k, 2}^{*} h_{k, 4}\right)\right)$ for $\mathrm{S}_{2}$.

Factually, according to the design of (21) and (23), the precoder matrix is not unique. Here, only if any pair of the four transmitted antennas rotates the phase by the corresponding $\theta$, the precoder is just feasible.

Extend to other transmit antenna number, the design is still effective. Take an example for $N_{t}=5$, the space-time coding matrix can be got by cancelling the three rows of MDC-STBCs with $N_{t}=8$ :

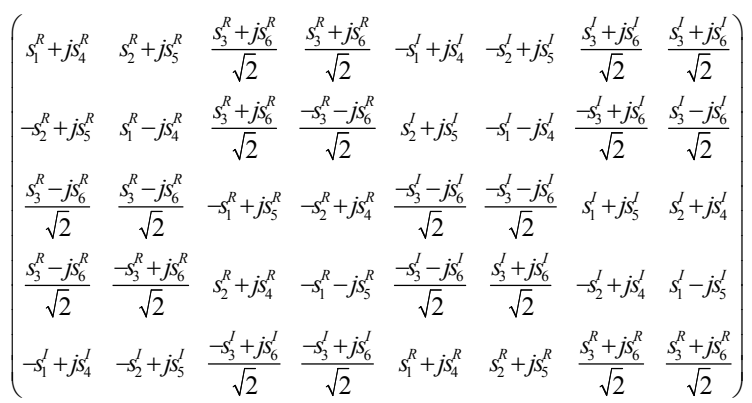

Then for it, the optimal precoder should be

$$
\mathbf{P}=\left[\begin{array}{lllll}
1 & & & & \\
& 1 & & & \\
& & 1 & & \\
& & 1 & \\
& & & e^{j \theta}
\end{array}\right] \text {, where } \theta=-\angle\left(\sum_{k=1}^{N_{r}}\left(h_{k, 1}^{*} h_{k, 5}\right)\right) .
$$

Now, we notice that the precoder only needs a parameter $\theta$ factually for these space-time coding matrix. When the system is channel-reciprocal, such as TDD system with low moving speed, we can easily get the channel coefficients to calculate the value of $\theta$ and generate the precoder matrix. When the channel-reciprocity does not hold, the index of a quantified $\theta$ can be fed back from the receiver to the transmitter. The proposed precoder is still valid. Only when the feedback bits are very limited, the quantification error isn't neglectable, the receiver cannot employ the HSD detection, but the SSD detection. However, the performance advantage due to the interference partly-cancellation after precoding still exists.

\section{Simulation Results}

In this section, we testify the performance of the proposed precoding scheme for MDC-STBC by simulation. Table II gives the simulation parameters. Because MDCSTBCs only obtain the full diversity gain when CR is employed [5], we use the MDC-STBC in [5] with CR as the reference scheme. And for the precoded MDC-STBC, both the ideal feedback and the quantified feedback with different feedback bits are considered in the simulation. For $m$-bit feedback scheme without feedback delay, the phase $\theta$ is quantified as $\left[0, \pi / 2^{m}, \cdots,\left(2^{m}-1\right) \pi / 2^{m}\right]$. 
TABLE II. SIMULATION PARAMETERS

\begin{tabular}{|c|c|}
\hline$N_{t} \times N_{r}$ & $4 \times 1$ \\
\hline Channel & Single-path Rayleigh channel \\
\hline Modulation & QPSK \\
\hline Detection algorithm & $\begin{array}{c}\text { ML (SSD for system without precoder; } \\
\text { HSD for that with precoder) }\end{array}$ \\
\hline
\end{tabular}

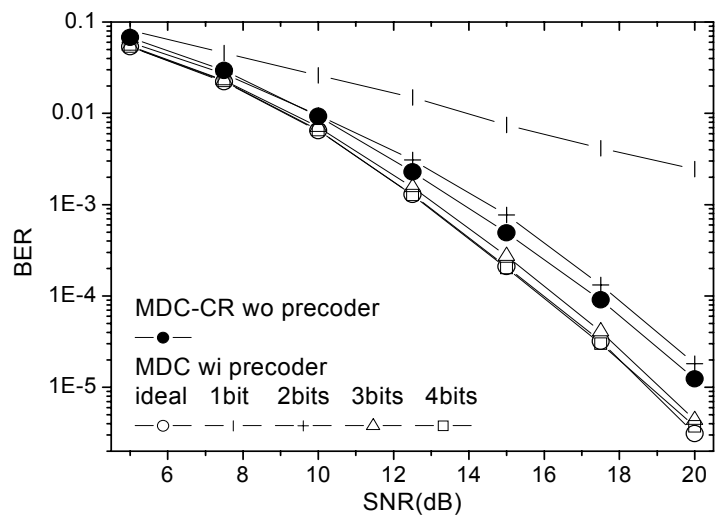

Figure 2. Performance comparison of precodered MDC-STBCs with different feedback bits

From Fig.2, we can see:

(1) The precoded MDC schemes with ideal feedback has the same slope to MDC-CR, that is the same diversity gain. The equivalent channel matrix in model (24) is a diagnal matrix with enties $r$, which be expressed as (14-i). That means this channel can offer the full diversity order and the maximum coding gain according to the lattice theory [16]. The gap between the curve of ideal feedback scheme and that of MDC-CR is just the earned extra coding gain due to the reconstructive perfect channel matrix by precoder.

(2) When the feedback of $\theta$ is not ideal, the coding gain will be partly lost. But the loss for the schemes of 3 bits and 4 bits feedback is small. For the scheme with 1 bit scheme, the precoder is meaningless due to the large quantification error.

Then considering the tradeoff between the performance and the quantity of feedback bits, 3 bits feedback is preferred. That is to say, comparing with the MDC-STBC scheme with $\mathrm{CR}$, a precoder with 3 bits feedback can avoid the CR operation and exploit the extra $1 \mathrm{~dB}$ coding gain, while leading to a simpler decoder.

\section{CONCLUSION}

In this paper, we focus on the precoding scheme for MDC-STBCs. After introducing the linear dispersion form for STBC, we explained how to construct an MDC-STBCs for the system with arbitrary antenna number in detail. Just for these MDC-STBCs, a precoding scheme was proposed, in which the precoder is a diagonal matrix with a phase parameter and designed to cancel the interference between to the real part and the imaginary part of the transmitted information symbols. The analysis of the decoding complexity and the ML decoding metric showed the employment of precoder make the decoding half-symbol decodable, while keeping the full diversity gain and maximum coding gain. Considering the issues of the limited feedback in the practical systems, we gave the performance comparison of the precoded schemes with the different feedback bits. Simulation results show that only 3 bits are enough for the precoder of MDC-STBCs with $N_{t}=4$ and the precoded scheme has a $1 \mathrm{~dB}$ gain than the scheme with full diversity gain by constellation rotation.

\section{ACKNOWLEDGMENT}

This work is supported by National Key Technology R\&D Program of China under Grant 2009ZX03007-002-03, and by the Research Fund for the Doctoral Program of Higher Education 20090005120002.

\section{REFERENCES}

[1] V.Tarohk etc., "Space-time block codes from orthogonal designs", IEEE Trans. Inf. Theogy, Vol.45, No.5, 1999, pp.1456-1467.

[2] Jafakhamij H. "A quasi-orthogonal space-time block codes", IEEE Trans on Communications, Vol.49, No.1, 2001,pp.1-4.

[3] N.Sharma and C.B.Papadias, "Improved quasi-orthogonal codes through constellation rotation", IEEE Trans. on Commun., Vol.51,No.3,2003, pp.332-335.

[4] Zafa Ali Khan and B.Sundar Rajan, "Space-time block codes from co-ordinate interleaved orthogonal design", ISIT 2002,pp.275.

[5] C. Yuen, Y. L. Guan, and T. T. Tjhung, "Quasi-orthogonal STBC with minimum decoding complexity: Futher results" IEEE WCNC, ,2005, pp.483-448.

[6] H.Wang etc., "On Optimal Quasi-Orthogonal Space-Time Block Codes with Minimum Decoding Complexity", ISIT,2005, pp.4-9.

[7] D.N.Dao, C.Tellambura, "Quasi-orthogonal STBC with minimum decoding complexity: performance analysis, optimal signal transformations, and antenna selection diversity", IEEE Trans. on. Comm, Vol.56, No.6, 2008,pp.849-853

[8] K.Sanjay and R.B.Sundar "Maximum-rate, Minimum-DecodingComplexity STBCs from Clifford Algebras", 2007, available at https://arxiv.org/pdf/0712.2371.

[9] D.J.Love, R.W.Heath., "Limited feedback unitary precoding for orthogonal space-time block codes", IEEE Trans. on Signal Processing, Vol.53, No.1,2005,pp.64-73

[10] Abdelkader Medles, Angeliki Alexiou, "New Design for Linear Precoding over STBC in the Presence of Channel Correlation", IEEE Trans. on Wireless Commu., Vol.6, No.4, 2007, pp.1203-1207

[11] X.F.Liu, F.L.FU, "Optimal Linear Precoding Design Based on the Quasi-Orthogonal STBC", Journal of Electronics and Information Technology, Vol.30, No.2, 2008, pp.401-403

[12] Rong Ran, Janghoon Yang and Dongku Kim, "Multimode precoder design for STBC with limited feedback in MIMO based wireless communication system", IEICE Electron. Express, Vol.6, No.3, 2009, pp. $173-179$

[13] B.Hassibi, B.M.Hochwald, "High-rate codes that are linear in space and time", IEEE Trans. Inform. Theory, Vol. 48, No. 7,2002,pp.18041823.

[14] K.J.Lu, S.L.Su and X.G.Xia, "closed-form designs of complex orthogonal space-time bolck codes fo rate $(\mathrm{k}+1) /(2 \mathrm{k})$ for $2 \mathrm{k}-1$ or $2 \mathrm{k}$ transmit antennas", IEEE Trans. Inform. Theory, Vol.51, 2005, pp.4340-4347.

[15] A. M. Tulino and S. Verdu, "Random Matrix Theory and Wireless Communications", Foundations and Trends in Communications and Information Theory. Now Publishers Inc, 2004.

[16] V. Tarokh, A. Vardy and K. Zeger, "Universal bound on the performance of lattice codes," IEEE Trans. on Inform. Theory, Vol. 45, No. 2, 1999,pp. 670-681. 